\title{
Dissipative trapped-electron instability in quasihelically symmetric stellarators
}

\author{
T. Rafiq and C. C. Hegna \\ Engineering Physics Department, University of Wisconsin, Madison, Wisconsin 53706-1609
}

(Received 3 March 2006; accepted 24 March 2006; published online 7 June 2006)

\begin{abstract}
The linear electrostatic dissipative trapped-electron mode is investigated in a quasihelically symmetric (QHS) stellarator and a configuration whose symmetry is spoiled by the addition of a mirror contribution to the magnetic spectrum. The effect of the trapped electrons is accounted for using the drift kinetic equation with an energy-dependent Krook collision operator and an effective collision frequency giving the rate of detrapping. The ballooning mode formalism and Wentzel-Kramers-Brillouin type boundary conditions are used to solve an eigenvalue problem for a drift wave equation with nearly adiabatic electrons in a fully three-dimensional magnetohydrodynamic equilibria. The trapped-electron growth rate is calulated using a perturbative approach. Multiple classes of helically localized and toroidally localized eigenfunctions in the ballooning space are calculated. The results of the QHS configuration is compared and contrasted with the results of the mirror configuration. The helically trapped modes are found to be most destabilizing. In both configurations the magnitude of the linear growth rates are comparable, crudely indicating the same level of anomalous flux as has also been observed in the edge region of experiments. (C) 2006 American Institute of Physics. [DOI: 10.1063/1.2204444]
\end{abstract}

\section{INTRODUCTION}

In the presence of $|\mathbf{B}|$ variation, trapped particle orbits are present in toroidal configurations. In tokamaks, for example, particles follow a helical magnetic field, and thus alternately experience strong (in the inner major radius region) and weak (in the outer major radius region) magnetic field strength $|\mathbf{B}|$ produced by the $1 / R$ dependence of the toroidal magnetic field. However, in stellarators, the helical ripple in $|\mathbf{B}|$ due to stellarator fields results in additional particle trapping mechanisms. When collisions are infrequent, trapped particles are not able to establish an exact Boltzmann distribution in the presence of electrostatic fluctuations, even for relatively low frequency perturbations. The existence of trapped particles is not only the origin of neoclassical diffusion, but is also the source of various drift wave instabilities. These are mainly classified into two categories: collisionless and dissipative trapped particle instabilities. The collisionless trapped particle instabilities are driven by magnetic curvature drift. ${ }^{1}$ On the other hand, the dissipative trapped-electron instability is similar to collisional drift wave in that the excitation depends on collisions. The instability on which we concentrate is the dissipative trapped-electron instability. It is speculated to be a damaging instability in toroidal systems. It is difficult to suppress these instabilities and can cause much anomalous diffusion. ${ }^{2}$ These instabilities have been first studied by Kadomstev and Pogutse ${ }^{3}$ and experimental evidence of these modes has also been found in linear plasma devices. ${ }^{4}$

Substantial efforts have been made to understand microinstabilities in tokamak geometries. However, very limited work has been published on these instabilities in stellarators and even less has been reported on modes driven by trapped electrons. $^{5-9}$ The quasihelically symmetric stellarator $(\mathrm{QHS})^{10,11}$ has a helical axis of symmetry closely approxi- mating the helical symmetry present in a straight stellarator. By restoring a direction of symmetry to a toroidal stellarator, the quasihelical stellarator solves one of the fundamental disadvantages of conventional stellarators: the poor neoclassical transport in the low collisionality regime. The helically symmetric experiment (HSX) ${ }^{12}$ is optimized to exhibit quasihelical symmetry. This is apparent in the $|B|$ spectrum of Fig. 1 (left) by the dominant helical mode $(n=1, m=1)$. To make a comparison to a conventional stellarator, the quasisymmetry can be broken with the addition of a $(n=1, m=0)$ toroidal mirror term. The spectrum of this mirror configuration is also shown in Fig. 1 (right). This mirror mode configuration increases the neoclassical transport and parallel viscous damping by orders of magnitude (back to the level of conventional stellarators) without appreciable changes in the volume, rotational transform, magnetic well depth, or ideal local magnetohydrodynamic (MHD) stability limit. Since neoclassical transport is optimized in QHS, it is likely that drift waves that are destabilized by a population of trapped electrons in the present operational regime of $\operatorname{HSX}\left(\nu_{*_{e}} \ll 1, T_{e} / T_{i}\right.$ $\gg 1, \eta_{i}<1, \eta_{e} \geqslant 1$ ), will become a dominant source of transport. The objective of the present paper is to report a study of dissipative trapped-electron modes (DTEM) in the QHS stellarator and to compare and contrast the results with the mirror mode configuration, which has high neoclassical transport.

The paper is organized as follows: In Sec. II, the collisionless drift wave equation for three-dimensional magnetic configurations is presented and a formula for the growth rate due to a dissipative trapped-electron mechanism is derived using a perturbative approach. A cold ion response is assumed, consistent with the operational regimes of HSX. In Sec. III, numerical results for the frequency, growth rate, and eigenfunctions are presented for QHS and mirror configura- 


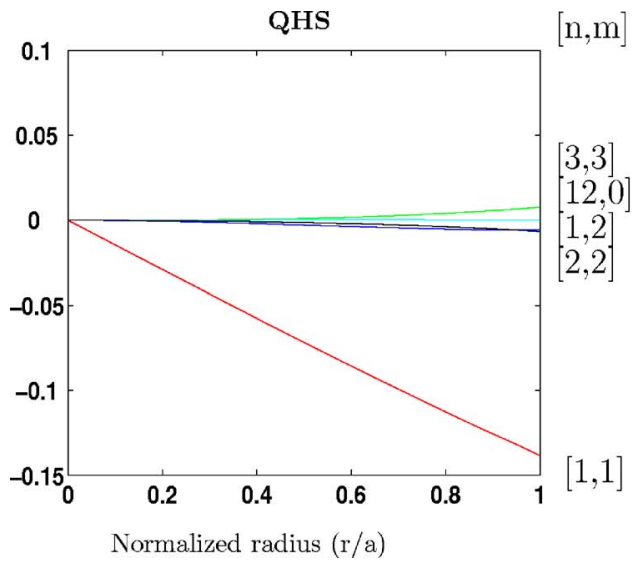

tions. Finally, a summary is given in Sec. IV.

\section{TRAPPED-ELECTRON MODES}

The dissipative trapped-electron instability is derived by solving the Vlasov-Maxwell equations in the electrostatic limit. The trapped electrons cannot move freely along the field line and their motion is described principally by their $\mathbf{E} \times \mathbf{B}$ drift across the field. The perturbed electron distribution function is

$$
f_{e 1}=\frac{|e| \phi f_{e 0}}{T_{e}}+g_{e}
$$

where $g_{e}$ is the trapped-electron part and is proportional to the bounce averaged potential $\langle\phi\rangle=\left(\int d t_{b} \phi\right) / \int d t_{b}$, and $f_{e 0}$ is the unperturbed Maxwellian distribution function. The unperturbed electrons are free to move along the field line, are known as passing particles, and are assumed to follow a Maxwellian at potential $\phi$. The bounce time $\tau_{b}=\int d t_{b}$ is the time for a trapped particle to complete one orbit along a field line. If the particle is scattered out of the trapping region before it has completed a banana type single bounce orbit, it cannot be regarded as a trapped one. Thus, if $\nu_{e f} \tau_{b} \gg 1$, trapping effects are negligible. Here we introduce $\nu_{e f}=\nu_{e i} / \epsilon(\epsilon$ $=B_{\max }-B_{\min } / B_{0}$ ) being the effective collision frequency for changing the direction of the velocity of a trapped particle. The quantity $\nu_{e f} \tau_{b}$ provides a measure of the collisionality of the plasma. The trapped particle effects will play an important role in the limit $\nu_{e f} \tau_{b} \ll 1$. The connection length of the helical field ripples in stellarators $\left(L_{c}=R /|N-m \iota|\right)$ is typically much shorter than the connection length of toroidal magnetic well in tokamaks $\left(L_{c} \sim q R\right)$, therefore the electron collisionality for helical trapping is proportionally lower than the toroidal collisionality at the same temperature and density. For the electrons, the condition $\nu_{e f} \tau_{b} \ll 1$ required for helical DTEMs can be fulfilled even at modest plasma parameters. $^{6}$

The equation for $g_{e}$ with the effect of detrapping is described by the Vlasov equation with an energy dependent Krook collision operator and an effective collision frequency, which gives the rate of detrapping. Using the Rutherford and Frieman approach ${ }^{13}$ and neglecting the finite Larmor radius (FLR) effects, we may write an equation for the $g_{e}$ as

$$
\left(-i \omega+\nu_{e f}+i \omega_{d e}+i \mathbf{V}_{\|} \hat{e} \cdot \nabla\right) g_{e}=\left(1-\frac{\omega_{* e}^{T}}{\omega}\right) \frac{i|e| \omega \phi}{T_{e}} f_{e 0},
$$

where $\omega_{*_{e}}^{T} \equiv \omega_{* e}\left[1+\eta_{e}\left(E / T_{e}-1.5\right)\right]$ is the velocity dependent electron diamagnetic drift frequency with $\eta_{e}$ $=d \ln T_{e 0} / d \ln n_{e 0}, E=m v^{2} / 2$ is the electron energy, and $\omega_{d e}$ is the electron $\nabla B$ and curvature drift frequency. The effective collisions frequency can be written as

$$
\nu_{e f}=\frac{\nu\left(v=v_{e}\right)}{\epsilon}\left(\frac{v_{e}}{v}\right)^{3},
$$

where $\nu\left(v=v_{e}\right)$ is the collisional detrapping frequency at the thermal velocity.

The trapped particle effects are significant in obtaining the particle response to oscillating the perturbed electric fields only in the case when oscillation frequency $\omega$ satisfies $\omega \tau_{b} \ll 1$. Hence we may impose the restriction

$$
\omega_{b} \sim\left|\mathbf{v}_{\|} \hat{e} \cdot \nabla\right|>\omega, \omega_{* e}, \nu_{e f}, \omega_{d e} .
$$

Then, to lowest order, Eq. (2) reduces to

$$
\left(\mathbf{v}_{\|} \hat{e} \cdot \nabla\right) g_{e}=0,
$$

and, consequently, $g_{e}$ is constant along the field line. To next order, after taking the bounce average of Eq. (2), an equation can be written as

$$
g_{e}=i \frac{|e| f_{e 0}}{T_{e}}\left(\frac{\omega-\omega_{* e}\left[1+\eta_{e}\left(E / T_{e}-1.5\right)\right]}{\nu_{e f}}\right)\langle\phi\rangle .
$$

The total (trapped and untrapped) perturbed electron number density can be obtained by integrating Eq. (1) over velocity space with $g_{e}$ given by Eq. (6). This yields the expression

$$
\begin{aligned}
\frac{\delta n_{e}}{n_{e}}= & \frac{e}{T_{e}}\left(\phi-i \frac{2}{\nu_{e f} \sqrt{\pi}}\left(\omega_{*_{e}}-\omega\right.\right. \\
& \left.\left.+1.5 \eta_{e} \omega_{*_{e}}\right) \int \frac{B d \Lambda}{\Lambda^{3 / 2} \sqrt{\Lambda-B}}\langle\phi\rangle\right),
\end{aligned}
$$

where the electron response is obtained by changing the variables from $v_{\perp}=\sqrt{(2 / m) B E / \Lambda}$ and $v_{\|}=\sqrt{(2 E / m)(1-B / \Lambda)}$ to $E$ and $\Lambda=E / \mu$ as in Ref. 7. Equation (7) is also correct for 
passing electrons in this regime since $\langle\phi\rangle$ vanishes, yielding a Boltzmann response. It is noted from Eq. (7) that the velocity dependences of the Coulomb collisions of the electrons and magnetic trapping are crucial to the instability. Moreover, Eq. (7) shows that the nonadiabatic response of the electron is directly proportion to $\eta_{e}$ and inversely proportional to $\nu_{e f}$. The collisions obviously strive to decrease the effect of trapping.

In the following, we derive the ion response with frequency in the regime $\omega_{b i} \ll \omega \ll \omega_{b e}$. In this case, the electron response is given by Eq. (7). As the wave phenomenon takes place on a time scale much shorter than the ion bounce time, the ion trapping effect will be negligible. Since the waves will be fast, i.e., $\omega / k_{\|} \gg V_{t i}$ for the ions, they cannot establish a Boltzmann distribution, so their contribution is given by the linearized equation of continuity and momentum, yielding

$$
\begin{aligned}
& \left(\frac{c_{s}^{2}}{\omega^{2}}\left(e_{\|} \cdot \nabla\right)^{2}-\rho_{s}^{2} \nabla_{\perp}^{2}-\left(\frac{1}{i \omega}\right) \vec{v}_{*} \cdot \nabla_{\perp}-\left(\frac{1}{i \omega}\right) \vec{v}_{D} \cdot \nabla_{\perp}\right) \frac{e \phi}{T_{e}} \\
& +\frac{\delta n_{i}}{n_{i}}=0 .
\end{aligned}
$$

The terms in Eq. (8) correspond to the cold ion dynamics for ion-sound, polarization drift, $\mathbf{E} \times \mathbf{B}$ drift written in terms of the diamagnetic velocity $\vec{v}_{*}$, and curvature drift $\vec{v}_{D}$ (from the divergence of $\mathbf{E} \times \mathbf{B}$ velocity in a curved field), respectively. Using the quasineutrality condition, ballooning mode formalism ${ }^{14}$ and ignoring the trapped-electron response in Eq. (7), the stable electrostatic eigenmode equation can be written as (see Ref. 15 for details)

$$
\frac{d^{2} \Phi}{d \zeta^{2}}+U(\zeta, \Omega) \Phi=0
$$

where $\Phi(\zeta)$ is the eigenfunction and $U(\zeta, \Omega)$ is the effective potential given as

$$
\begin{aligned}
U(\zeta, \Omega)= & -\left(\frac{J B}{q \bar{R} \dot{\psi}}\right)^{2}\left\{\left(\Omega_{*}+\Omega_{d}\right) \chi \Omega\right. \\
& \left.-\left[1+\left(\frac{\chi B_{0}}{B}\right)^{2}\left(\hat{\mathbf{k}}_{\perp} \cdot \hat{\mathbf{k}}_{\perp}\right)\right] \Omega^{2}\right\} .
\end{aligned}
$$

Here,

$$
\begin{aligned}
& \Omega_{*}=\Omega_{*}(s)=-\frac{2}{\epsilon_{n} \vec{a}}, \quad \epsilon_{n}=\frac{L_{n}}{\bar{R}}, \quad \Omega=\frac{\bar{R} \omega}{c_{s}}, \\
& \chi=\epsilon^{-1} \frac{q \rho_{s o}}{\bar{a}} \frac{\partial S}{\partial \alpha}, \quad c_{s}=\sqrt{\frac{T_{e}}{m_{i}}}, \quad \hat{\phi} \equiv \frac{e \phi}{T_{e}}, \\
& \Omega_{d}=\Omega_{d}(s, \alpha, \zeta)=B_{0} \bar{R}\left(\frac{\mathbf{B} \times(\boldsymbol{\kappa}+\nabla \ln B)}{B^{2}}\right) \cdot \hat{\mathbf{k}}_{\perp}, \\
& \rho_{s o}=\frac{c_{s}}{e B / m_{i}},
\end{aligned}
$$

where, $\epsilon$ is the Wentzel-Kramers-Brillouin (WKB) expansion parameter, $S$ is the ballooning mode eikonal, $L_{n}$ is the density scale length, $T_{e}$ is the electron temperature, and $c_{s}$ is the ion sound speed with electron temperature. The perpendicular wave number is given as

$$
\begin{aligned}
& \mathbf{k}_{\perp}=\epsilon^{-1} \frac{\partial S}{\partial \alpha}\left\{\nabla \alpha+\Theta_{k} \dot{q} \boldsymbol{\nabla} s\right\}=\epsilon^{-1} \frac{q}{\bar{a}} \frac{\partial S}{\partial \alpha} \hat{\mathbf{k}}_{\perp}, \text { with } \\
& \Theta_{k}=\frac{\partial S / \partial q}{\partial S / \partial \alpha},
\end{aligned}
$$

where the normalized perpendicular wave vector is

$$
\begin{aligned}
\hat{\mathbf{k}}_{\perp}= & \hat{\mathbf{k}}_{\perp}\left(s, \alpha, \zeta, \theta_{k}\right)=\frac{\bar{a}}{q}\left[\boldsymbol{\nabla} \zeta-q \boldsymbol{\nabla} \theta-\left(\frac{\zeta-\zeta_{0}}{q}\right.\right. \\
& \left.\left.-\theta_{k}\right) \dot{q} \boldsymbol{\nabla} s\right]
\end{aligned}
$$

with $\theta_{k}=\Theta_{k}-\theta_{0}$ and $\dot{q}=d q / d s$. Equation (9) describes the universal electron drift modes with adiabatic electrons. The drift mode may become unstable if the electron response is not pure Boltzmann, as described, for example, by the dissipative term in Eq. (7). The ballooning mode formalism and WKB type boundary conditions ${ }^{16,17}$ are used to solve an eigenvalue problem for a drift wave equation with adiabatic electrons in a fully three-dimensional hydrodynamic equilibria. The equilibria is computed using the VMEC code ${ }^{18}$ with fixed boundary conditions for QHS and mirror mode configurations and for a set of 98 magnetic surfaces. The VMEC coordinate system is not a straight field line coordinate system. Thus, before solving the drift wave Eq. (9), we first transform the equilibrium to Boozer coordinates. ${ }^{19}$ Then the contravariant and covariant basis vectors are constructed for each flux surface and used to calculate magnetic field, the magnetic field line curvature, the local magnetic shear, and the derivatives of the magnetic field in a fully threedimensional configuration. Details are given in Ref. 17.

To determine the growth rate for a DTEM, we can write Eq. (9) including trapped-electron response as

$$
L(\omega) \Phi=R(\omega) \Phi,
$$

where $L(\omega) \Phi$, is the left-hand side of Eq. (9) with $L(\omega)$ being the linear operator. The electron trapping terms are written $R(\omega) \Phi$, thus defining another linear operator $R(\omega)$. A perturbation theory is used to show ${ }^{5,13}$ a change in frequency:

$$
\delta \omega=i \frac{\int d \zeta / B^{2} \Phi^{*} R \Phi}{\int d \zeta / B^{2} \Phi^{*}(d L / d \omega) \Phi} .
$$

Using the solution of Eq. (9) with an adiabatic electron response, we calculate $\phi(\zeta)$ and $\omega_{0}$, and then perturbatively using Eq. (11) we calculate a change in frequency, $\delta \omega$, due to nonadiabatic electron contribution, ${ }^{5}$ as

$$
\frac{\delta \omega}{\omega_{0}}=i \frac{4}{\nu_{e f} \sqrt{\pi}}\left(\omega_{*_{e}}-\omega_{0}+1.5 \eta_{e} \omega_{*}\right) \frac{H_{1}}{H_{2}},
$$

where 

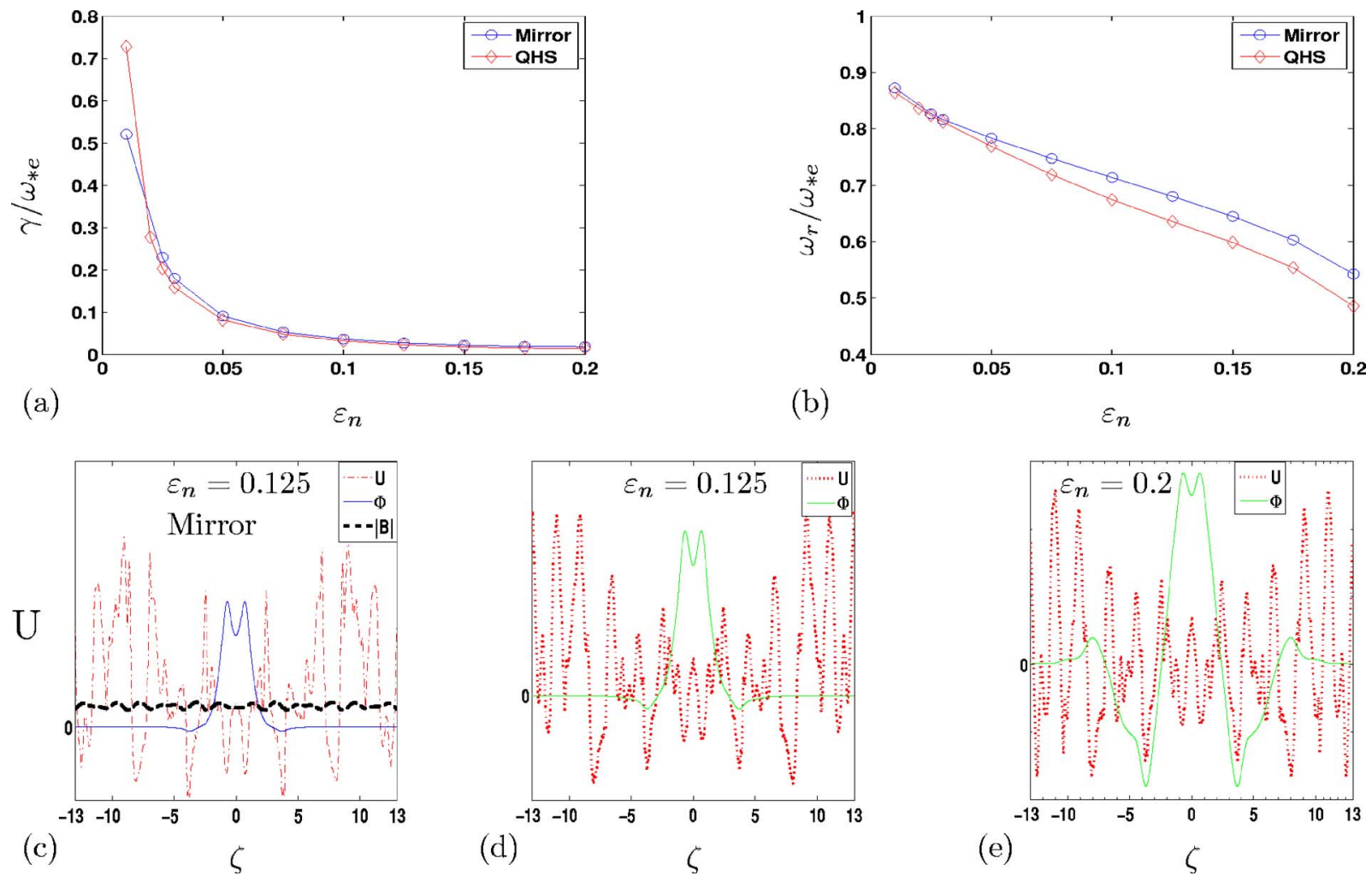

(b)

$\varepsilon_{n}$

FIG. 2. (Color online) (a) Normalized growth rate, $\gamma / \omega_{*}$, as a function of $\varepsilon_{n}=L_{n} / R$. (b) Normalized real frequency, $\omega_{r} / \omega_{*}$, for DTEMs as a function of $\varepsilon_{n}$ for the QHS and mirror configurations. The parameter values used are $b=0.1, \theta_{k}=0.0$, along the field line passing through $\theta_{0}=0$, $\zeta_{0}=0$ on the flux surface $s=0.8980$. Some of the collisionless drift wave eigenfunctions $(\Phi)$ and their corresponding effective potentials $(U)$ and $|B|$ as function of $\zeta$ are shown in (c)-(e). As the density profile is broadened the structure of the effective potential changes, the eigenfunction becomes extended, with a corresponding decrease in growth rate and real frequency. The maximum growth rate corresponds to the mode that is localized to the smallest number of helical wells and has the steepest density profile. The drift wave frequency deviates somewhat from $\omega_{*}$ due to the effects of grad $B /$ curvature drifts, finite ion Larmor radius, parallel ion motion, electron temperature gradients, and trapped electron. Peaking of the density profile raises the real frequency relative to $\omega_{*}$.

$$
H_{1}=\sum \int_{B_{\min }}^{B_{\max }} \frac{d \Lambda}{\Lambda^{3 / 2}} \frac{\left|\int_{\zeta_{-}}^{\zeta_{+}} d \zeta \Phi_{0} / B \sqrt{\Lambda-B}\right|^{2}}{\int_{\zeta_{-}}^{\zeta_{+}} d \zeta / B \sqrt{\Lambda-B}}
$$

and

$$
H_{2}=\int_{0}^{\infty} \frac{d \zeta}{B^{2}}\left|\Phi_{0}\right|^{2} \frac{B_{0}}{B}\left(2 \kappa_{\perp}^{2} \rho_{s}^{2} \chi^{2}-\chi \frac{\omega_{*_{e}}+\omega_{d}}{\omega_{0}}+2\right) .
$$

Here $\omega_{0}$ and $\Phi_{0}$ are the real frequency and the eigenfunctions of the collisionless drift wave Eq. (9). The integration over $\zeta$ (along the field line) is carried out between turning points, and over the particle energy $\Lambda=E / \mu$ between maximum and minimum values of $\mathbf{B}$ along the field line in one trapping interval. A summation has been carried out on all the trapping intervals in $H_{1}$.

Stellarators support at least two classes of trapped particles: those that are helically trapped and those that are toroidally trapped. The various classes of trapped particles interact quite differently with stellarator drift modes. The helically trapped particles couple strongly to helically induced modes with $\langle\Phi\rangle \approx \Phi$. These modes have a narrow extend along the field lines and, as a consequence, a broad radial extent. Moreover, a helically extended mode can couple to several sets of helically trapped particles. These modes span over several helical periods and couple to outgoing waves. Both type of eigenfunctions, helically localized as well as toroidally localized, are observed in ballooning space.

\section{NUMERICAL RESULTS AND DISCUSSION}

The variation of normalized growth rate and real frequency with the normalized density gradient length scale $\varepsilon_{n}$ is shown in Figs. 2(a) and 2(b) for the QHS and mirror configurations. Some of the collisionless drift wave eigenfunctions, $(\Phi)$ and their corresponding effective potentials $(U)$, and $|\mathbf{B}|$ as function of $\zeta$ are shown in Figs. 2(c)-2(e). Peaking of the density profile raises the real frequency and the growth rate in both cases due to the high degree of localization of the eigenfunctions in trapped particle regions. Moreover, as the density profile is broadened, the structure of the effective potential changes; the eigenfunction becomes extended, while the growth rate and real frequency decreases. The maximum growth rate in the QHS corresponds to the mode that is localized in the smallest number of helical wells and has a steep density profile. Altogether, the magnitude of the growth rate is found to be similar in both configurations except for cases with a peaked density profile. The smaller growth rate is found in the mirror case at the peaked density and is due to the structure of $|\mathbf{B}|$, which has a 


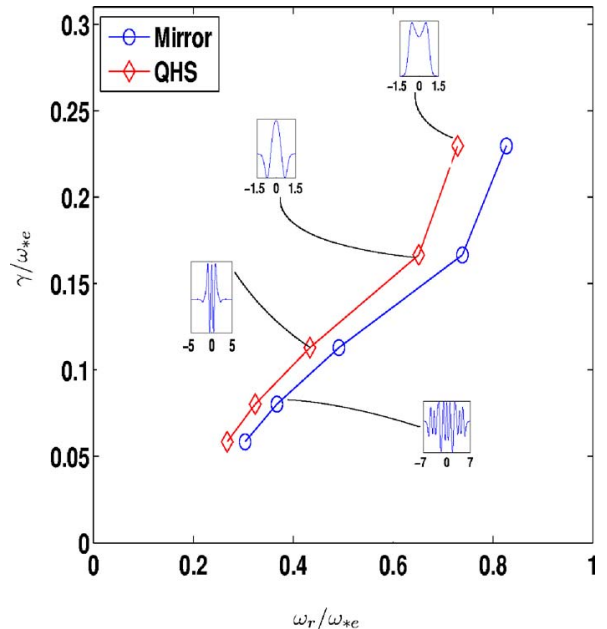

FIG. 3. (Color online) (a) Strongly and weakly localized eigenfunctions for $\varepsilon_{n}=0.025$. The other parameters are the same as used in Fig. 2. The localized eigenfunction is found to be the most unstable for the peak density profile.

relatively shallow helical well and slightly less bad curvature around $\zeta=0.0$, as shown in Figs. 5 and 6 of Ref. 15.

There can be several solutions to the drift wave equation for a fixed set of parameters. Both strongly localized and weakly localized modes with a different number of nodes in the eigenfunction are seen in both cases. These eigenfunctions are shown in Fig. 3 for the QHS and mirror cases, respectively. The most localized (helical) modes are found to be most destabilizing. This result has been shown in Ref. 9. While the neoclassical transport in the QHS case is significantly smaller than the mirror configuration, the magnitudes of the linear growth rates for the two configurations are comparable. Hence, one might anticipate comparable levels of anomalous transport in the two cases.

The variation of normalized growth rate and real frequency with $b=\chi_{\perp}^{2}\left|\hat{\mathbf{k}}_{\perp} \cdot \hat{\mathbf{k}}\right|_{\zeta=\zeta_{0}}$ is shown in Figs. $4(\mathrm{a})$ and $4(\mathrm{~b})$ for the QHS and mirror cases. Some of the collisionless drift wave eigenfunctions $(\Phi)$ and their corresponding effective potentials $(U)$, and $|B|$ as function of $\zeta$ are shown in Figs. 4(c)-4(e). In both cases, the growth rate of DTEM and the collisionless real frequency decreases with the increase of $b$. However, the stabilizing effects due to the FLR are found to be weak and even weaker in the QHS configuration. In the QHS case, as the eigenfunction becomes more and more localized, it will become very well trapped in the first helical well of $|\mathbf{B}|$ around $\zeta=0$, and shows a slightly larger growth rate.

In Fig. 5, the chosen field line is changed from $\theta_{0}=0.0$ to $\theta_{0}=\pi$, which falls on the inboard side of the symmetry point where local normal curvature is good in both QHS and mirror configurations. All other parameters are the same as used in Fig. 2. In both equilibria, localized modes are found to be stabilized in the region of good curvature and in the antihelical well of $|\mathbf{B}|$. This result contrasts the result using a
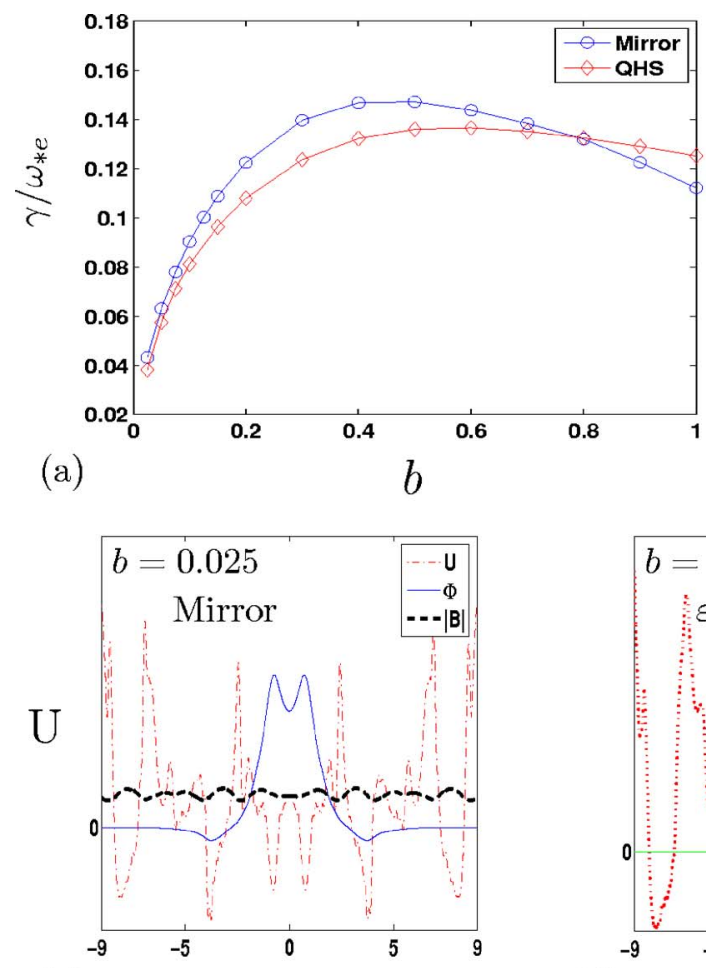

(c)

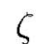

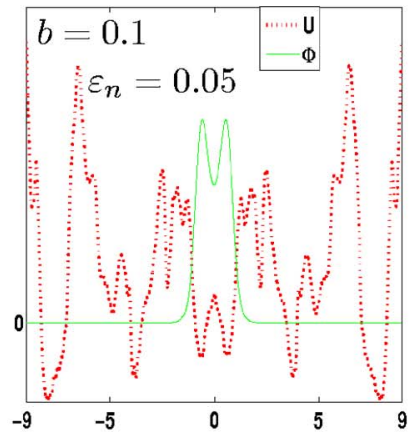

(d)

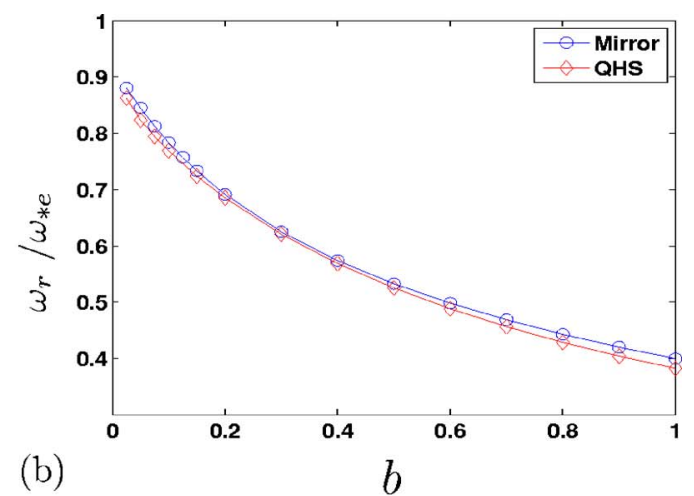

FIG. 4. (Color online) (a) Normalized growth rate, $\gamma / \omega_{* e}$, (b) real frequency, $\omega_{r} / \omega_{*_{e}}$ for DTEMs as a function of $b$ for the QHS and mirror configurations and for $\varepsilon_{n}=0.025$ and $\varepsilon_{n}=0.05$. The other parameter values used are the same as used in Fig. 2 . Some of the eigenfunctions $(\Phi)$ and their corresponding effective potentials $(U)$ and $|\mathbf{B}|$ as a function of $\zeta$ are shown in (c)-(e). It is found that as $b$ increases, eigenfunctions become more and more localized and trapped in the first helical well of $|\mathbf{B}|$ and effective potential. The growth rate of DTEM and the collisionless real frequency decreases with the increase of $b$. However, the stabilizing effect of $b$ altogether is found to be weak and even weaker in the QHS configuration. 

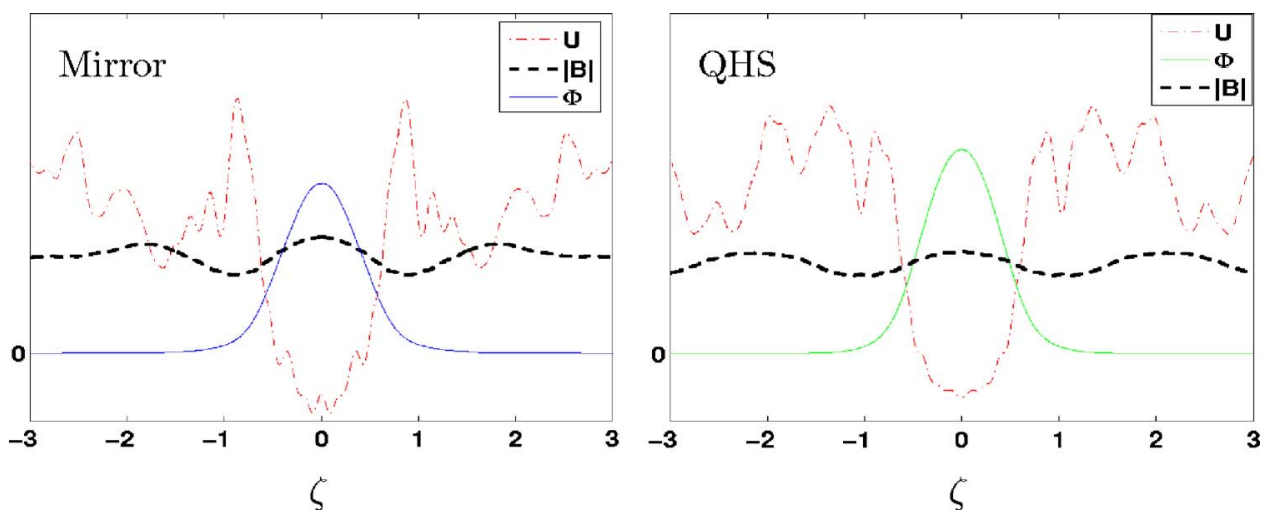

FIG. 5. (Color online) $U,(\Phi)$, and $|B|$ as a function of $\zeta$ along the field line passing through $\theta=\pi, \zeta_{0}=0$. The other parameters are the same as used in Fig. 2. Localized modes are found to be stabilized in the region of good curvature and in the antihelical well of $|\mathbf{B}|$ in both geometries. simple $i \delta$ model $^{15}$ where unstable drift modes are found both in good and bad curvature regions. A more realistic model for the dissipative mechanism allows for a more accurate treatment of the instability mechanism.

Finally, the DTEM growth rate and real frequency in the QHS configuration is calculated as a function of the magnetic surface label. For this calculation, the density and temperature profiles used are plotted in Fig. 6(f). This profiles closely model the actual profiles seen in HSX. Figure 6(e) shows that eigenfunctions at different magnetic surfaces. The DTEM is found to be marginally stable in the core and the edge is found to be more unstable due to the increasing value $\eta_{e}$ and decreasing value of electron temperature [see Fig. 6(c)] at a larger magnetic surface. This trend is similar to the experiment results where it has been observed that particle transport increases toward the edge. The stability of the core region is due to the $|\mathbf{B}|$ [see Fig. 6(d)], which has shallow helical wells relative to the edge and therefore yields a trapped-electron population that is too weak to excite a trapped-electron instability. The drift wave frequency in Fig. 6(b) deviates somewhat from $\omega_{*_{e}}$ due to the effects of $\operatorname{grad} B /$ curvature drifts, finite ion Larmor radius, parallel ion motion, electron temperature gradients, and the trappedelectron response.

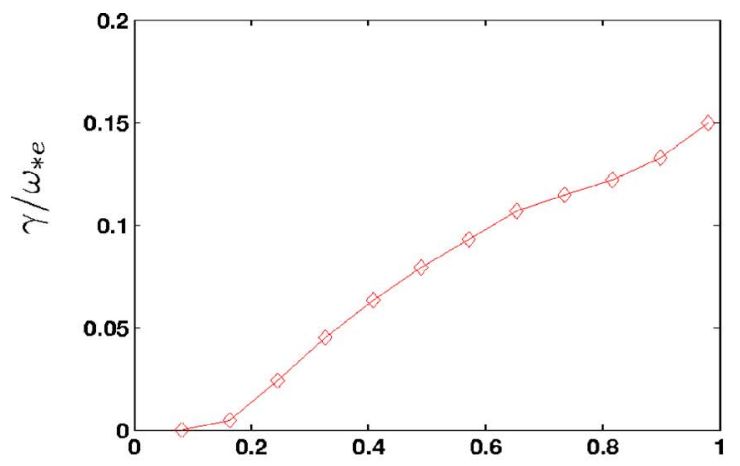

(a)

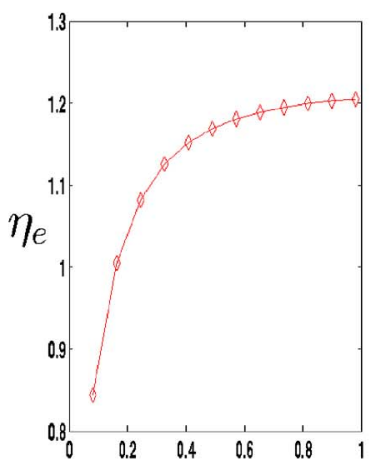

(c)

$S$ $s$

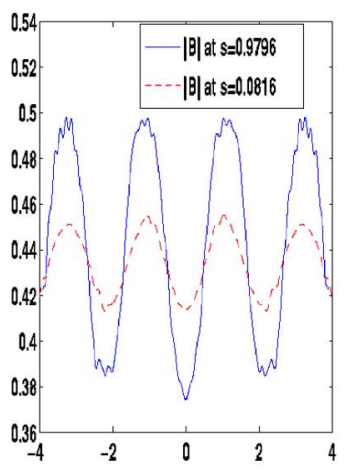

(d)

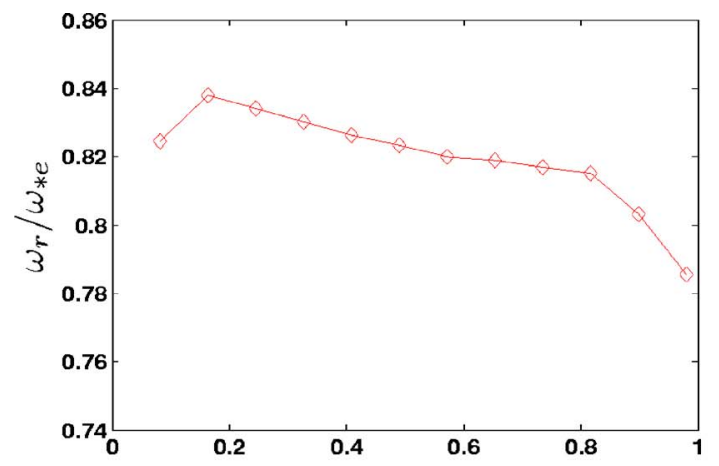

(b)

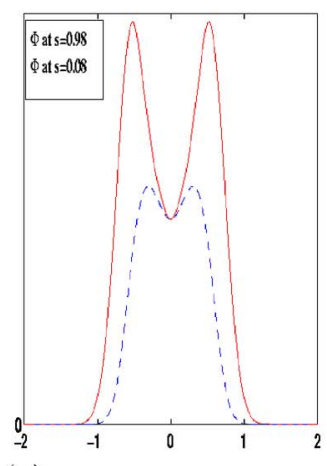

(e)

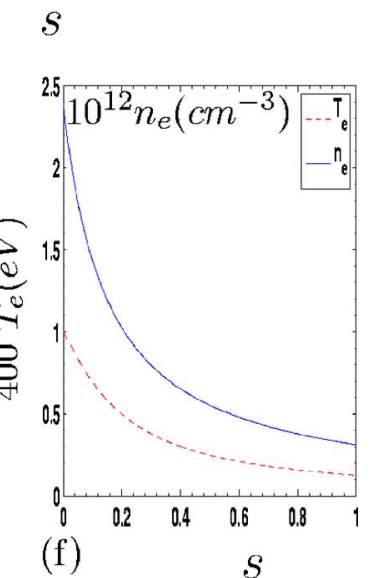

(f)

FIG. 6. (Color online) (a) Normalized growth rate, $\gamma / \omega_{*_{e}}$ and (b) real frequency, $\omega / \omega_{*_{e}}$, for DTEM as a function of $s$ for the QHS configuration. The parameter values used are $b=0.1, \theta_{k}=0.0$, along the field line passing through $\theta_{0}=0, \zeta_{0}=0$. The $\eta_{e}(s),|\mathbf{B}(\zeta)|, \Phi(\zeta)$, electron density, and temperature profiles are shown in (c)-(f). The plasma is marginally stable to the DTEM in the core and the edge is found to be more unstable. This is due to the profile of $\eta_{e}$, which increases toward the edge, and the electron temperature profile, which has small values at the edge. The stability of the core region is due to the structure of $|\mathbf{B}|$, which has shallow helical wells and therefore does not helically localize the eigenfunctions. 


\section{SUMMARY}

Dissipative trapped-electron modes (DTEM) is investigated in the electrostatic limit using ballooning mode formalism. For these modes, a shooting code technique is used and WKB type boundary conditions are applied. The threedimensional equilibria is calculated for a quasihelically symmetric (QHS) stellarator and a configuration whose symmetry is spoiled by the presence of a mirror contribution to the magnetic spectrum. These configurations are relevant to HSX experimental operation. The dependence of the drift wave spectrum of stable and unstable modes on various equilibrium parameters are investigated. The DTEM growth rate is calculated using a perturbative approach. Multiple classes of helically localized and toroidally localized eigenfunctions in the ballooning space are taken into account. The helically trapped modes are found to be most destabilizing. The magnitude of the growth rate is found to be similar in both configurations except for cases with a peaked density profile. The QHS has a helical axis of symmetry closely approximating the helical symmetry present in a straight stellarator. Because of this property the QHS results are consistent with the earlier published straight stellarator results. ${ }^{5}$ The observation that the most localized modes in the QHS configuration are the most unstable is consistent with the result reported in Ref. 9. The edge of the QHS configuration is found to be more unstable as compared to the center. This trend is similar to the QHS experiment results where it has been observed that transport increases toward the edge. While the neoclassical transport in the QHS case is significantly smaller than the mirror configuration, the magnitudes of the linear growth rates for the two configurations are comparable. Hence, one might anticipate comparable levels of anomalous transport in the two cases as has also been observed in the edge region of experiments.

\section{ACKNOWLEDGMENTS}

The authors thank Dr J. N. Talmadge and W. Guttenfelder for providing HSX equilibria data. This research was supported by the U.S. DOE under Grant No. DEFG0299E54546.

${ }^{1}$ B. B. Kadomtsev and O. P. Pogutse, Nucl. Fusion 11, 67 (1971).

${ }^{2}$ K. Miyamoto, Plasma Physics for Nuclear Fusion (MIT Press, Cambridge, MA 1989), p. 387.

${ }^{3}$ B. B. Kadomtsev and O. P. Pogutse, Sov. Phys. Dokl. 14, 470 (1969).

${ }^{4}$ S. C. Prager, A. K. Sen, and T. C. Marshall, Phys. Rev. Lett. 33, 692 (1974).

${ }^{5}$ N. Dominguez, B. A. Carreras, V. E. Lynch, and P. H. Diamond, Phys. Fluids B 4, 2894 (1992).

${ }^{6}$ M. G. Shats, J. H. Harris, K. M. Likin, J. B. Wilgen, L. R. Baylor, J. D. Bell, C. H. Ma, M. Murakami, K. A. Sarksyan, S. C. Aceto, T. S. Bigelo, G. L. Bell, R. J. Colchin, R. A. Dory, J. L. Dunlap, G. R. Dyer, A. C. England, R. C. Goldfinger, G. R. Hanson, D. P. Hutchinson, R. C. Isler, T. C. Jernigan, R. A. Langley, D. K. Lee, J. F. Lyon, A. L. Qualls, D. A. Rasmussen, R. K. Richards, M. J. Saltmarsh, J. E. Simpkins, K. L. Vander Sluis, and J. J. Zielinski, Phys. Plasmas 2, 398 (1995).

${ }^{7}$ A. Kendl and H. Wobig, Phys. Plasmas 12, 4714 (1999).

${ }^{8}$ G. Rewoldt, L. P. Ku, W. M. Tang, H. Sugama, N. Nakajima, K. Y. Watanabe, S. Murakami, H. Yamada, and W. A. Cooper, Nucl. Fusion 42, 1047 (2002).

${ }^{9}$ G. Rewoldt, L. P. Ku, W. M. Tang, and H. Sugama, Phys. Plasmas 12, $102512(2005)$.

${ }^{10}$ J. Nührenberg and R. Zille, Phys. Lett. A 129, 113 (1988).

${ }^{11}$ J. Nührenberg and R. Zille, Phys. Lett. A 114, 129 (1986).

${ }^{12}$ F. S. B. Anderson, A. F. Almagri, D. T. Anderson, P. G. Mathews, J. N. Talmadge, and J. L. Shohet, Fusion Technol. 27, 273 (1995).

${ }^{13}$ P. H. Rutherford and E. A. Frieman, Phys. Fluids 11, 569 (1968).

${ }^{14}$ R. L. Dewar and A. H. Glasser, Phys. Fluids 26, 3038 (1983).

${ }^{15}$ T. Rafiq and C. C. Hegna, Phys. Plasmas 12, 112505 (2005).

${ }^{16}$ M. Persson, J. L. V. Lewandowski, and H. Nordman, Phys. Plasmas 3, 3720 (1996).

${ }^{17}$ T. Rafiq, J. Anderson, M. Nadeem, and M. Persson, Plasma Phys. Controlled Fusion 43, 1363 (2001).

${ }^{18}$ S. P. Hirshman and O. Betancourt, J. Comput. Chem. 96, 99 (1991).

${ }^{19}$ A. H. Boozer, Phys. Fluids 25, 520 (1982). 